\title{
Fourier Analysis on a Hyperbolic Supermanifold with Constant Curvature
}

\author{
Martin R. Zirnbauer
}

Institut für Theoretische Physik, Universität zu Köln, W-5000 Köln, Federal Republic of Germany

Received October 24, 1990

\begin{abstract}
The Fourier inversion theorem is proved for a rank-one noncompact homogeneous space, the hyperbolic superplane. The proof makes use of some novel features of perfectly graded superspaces, which are not encountered in classical geometric analysis. An application to quasi-one-dimensional disordered one-electron systems is given.
\end{abstract}

\section{Introduction}

The theory of quantum transport and localization in disordered one-electron systems distinguishes between three universality classes, each being described by a statistical ensemble of Hamilton operators with local gauge invariance, which is either orthogonal, or unitary, or symplectic. Avoiding the replica trick used in the pioneering work of Wegner [15] and of Wegner and Schäfer [11], Efetov [5] showed how to calculate ensemble averages of products of Green's functions for each universality class, by means of a mapping onto nonlinear $\sigma$ models with super coset spaces $\mathbf{G} / \mathbf{K}$ for their target spaces. A fruitful advance in extracting physical information from these models has recently been made by Iida, Weidenmüller and Zuk [8]. Starting from the Landauer-Büttiker formula for the conductance [12], and using $S$-matrix techniques developed in the context of statistical nuclear reaction theory $[9,13]$, they mapped the problem of calculating the average conductance of a quasi-one-dimensional metallic system onto the problem described in the next paragraph.

With $e$ being the unit element of $\mathbf{G}$, let $o=e \mathbf{K}$ denote the origin of the space $\mathbf{G} / \mathbf{K}$ that is associated with the disordered conductor under consideration. The elements of $\mathbf{G} / \mathbf{K}$ are left cosets $g \mathbf{K}(g \in \mathbf{G})$, which are written $g \cdot o \stackrel{\text { def }}{=} g \mathbf{K}$, the operator "." denoting the transitive action of $\mathbf{G}$ on $\mathbf{G} / \mathbf{K}$ by left translation. Let $\mathbf{G} / \mathbf{K}$ be given its natural $\mathbf{G}$-invariant geometry, let $\boldsymbol{\Delta}_{\mathbf{G} / \mathbf{K}}$ denote the Laplace-Beltrami operator on $\mathbf{G} / \mathbf{K}$, and let $f(g \cdot o ; s)$ be the solution of the heat equation

$$
\partial_{s} f=\Delta_{\mathbf{G} / \mathbf{K}} f
$$


for a certain initial condition $f(g \cdot \sigma ; 0)=f_{0}(g \cdot o)$, which we do not specify here, except to say that it is determined by the coupling of the disordered conductor to external leads. The average conductance $\langle c\rangle$ is then given by

$$
\langle c\rangle=\int_{\mathbf{G} / \mathbf{K}} D g_{\mathbf{K}}|f(g \cdot o ; L / 2 \xi)|^{2},
$$

where $D g_{\mathbf{K}}$ is the $\mathbf{G}$-invariant Berezin measure on $\mathbf{G} / \mathbf{K}$, and $L / \xi$ the length of the system measured in units of a suitably defined correlation length $\xi$. Thus, in a qualitative manner of speaking, quantum mechanical transport in an ensemble of disordered conductors with orthogonal, unitary, or symplectic gauge symmetry, is related to diffusion on the corresponding supermanifold $\mathbf{G} / \mathbf{K}$. The relation becomes exact if the disordered system is described by the limit $N \rightarrow \infty$ of Wegner's $N$-orbital model [14]. Formulas of the type (0.2) exist not only for the mean value of the conductance but for any of its moments.

What makes the above formulation superior to earlier approaches [5] based on the Kubo formalism is the very high degree of group symmetry of Eq. (0.1). Although the average conductance has so far been calculated only in the limits $L / \xi \ll 1$ [8] and $L / \xi \gg 1$ [5], it is reasonable to believe that the solution to the problem posed by Eqs. (0.1) and (0.2) can be obtained in exact and rather explicit form for arbitrary values of $L / \xi$. Certainly, given the heat kernel $W$ on $\mathbf{G} / \mathbf{K}$, we have the formal solution

$$
\langle c\rangle=\int_{\mathbf{G} / \mathbf{K}} D g_{\mathbf{K}} \int_{\mathbf{G} / \mathbf{K}} D h_{\mathbf{K}} \bar{f}_{0}(h \cdot o) W\left(g^{-1} h ; L / \xi\right) f_{0}(g \cdot o) .
$$

(We mention in passing that heat kernels for a certain class of super Laplace operators have been calculated by Aoki [2].) However, we can go even further than in $(0.3)$, by using the Fourier transform $f \mapsto \tilde{f}$ and a generalization of the Plancherel formula to functions on $\mathbf{G} / \mathbf{K}$, to write

$$
\langle c\rangle=\int_{\mathbf{a}^{+*}}\left(\int_{\mathbf{K}} D k\left|\tilde{f}_{0}(\lambda, k)\right|^{2}\right) e^{-L / \xi\left(|\rho|^{2}+|\lambda|^{2}\right)} d \mu(\lambda) .
$$

Here $\mathbf{a}^{+*}$ is the dual of a positive Weyl chamber for $\mathbf{G} / \mathbf{K}, D k$ the invariant Berezin measure on $\mathbf{K},|\rho|^{2}+|\lambda|^{2}$ an eigenvalue of $\Delta_{\mathbf{G} / \mathbf{K}}$, and $d \mu(\lambda)$ the Plancherel measure. It turns out that the calculation of the Fourier transform of $f_{0}$ is quite simple for realistic disordered samples with a large number of scattering channels contributing to transport at the Fermi energy. Also, the integral over $\mathbf{K}$ is very easy to perform since $f_{0}$ is the component of a vector that transforms as the adjoint representation of $\mathbf{K}$. Therefore, from a purely practical point of view, the only difficult step in this approach is the construction of the Plancherel measure $d \mu(\lambda)$. On a rigorous level, however, one needs to justify the Plancherel formula, and this is a nonstandard mathematical problem as we shall now explain briefly.

The base manifold of Efetov's super coset space $\mathbf{G} / \mathbf{K}$ for each universality class is obtained by forming the direct product of two symmetric spaces, one compact and the other one noncompact. (The outer integral sign in (0.4) actually stands for both integrations and summations.) The theory of Fourier analysis on symmetric spaces has reached a highly developed level $[6,7]$ but, as a matter of fact, the techniques used for spaces of the compact and noncompact type are rather different. In the first case, one exploits the properties of roots and weights of a Cartan 
subalgebra, whereas in the second case one studies the asymptotic behavior of the spherical functions and is led to the introduction of Harish-Chandra's c-function. It is thus clear that a theory of Fourier analysis on Efetov's super coset spaces requires not only the generalization to supermanifolds, but also a considerable extension and unification, of classical theory.

Having established the general physical context, we now turn to the more specific subject of the present paper. We will solve the problem analogous to, but simpler than, (0.1) and (0.2) which is obtained by replacing Efetov's spaces by a rank-one homogeneous space $\mathbf{G} / \mathbf{K}$, referred to as the "hyperbolic superplane." This space shares with Efetov's spaces two prominent features: (i) perfect grading ${ }^{1}$ and (ii) noncompactness. From the author's experience, the nonlinear $\sigma$ model which has the hyperbolic superplane for its target space may in fact serve as a useful "toy model" for .studying diffusion and localization in disordered oneelectron systems.

To construct the hyperbolic superplane, we start from its base manifold, which is the noncompact symmetric space $\mathrm{H}_{2}=S O(2,1) / S O(2)$. $\mathrm{H}_{2}$ may be viewed as a real manifold with dimension two or, alternatively, as a complex manifold with (complex) dimension one. In either view, there exists a natural way of adding two real anticommuting coordinates (respectively one complex anticommuting coordinate) to extend $\mathrm{H}_{2}$ to a perfectly graded supermanifold. The two spaces so obtained differ from each other in their natural geometry, which is defined by the requirement of invariance under the corresponding extension of $S O(2,1)$ to a Lie supergroup. The space called the hyperbolic superplane and analyzed in the present paper is the first of these, i.e. the one obtained by viewing $\mathrm{H}_{2}$ as a real manifold. Being a homogeneous space with invariant geometry, it has constant curvature, as is anticipated by the title of the paper. It turns out that the second space, obtained by viewing $\mathrm{H}_{2}$ as a complex manifold, is not a suitable prototype for the study of disordered systems, as it has vanishing scalar curvature and therefore does not lead to "exponential localization" in the limit of strong disorder.

As the hyperbolic superplane has a noncompact symmetric space for its base manifold, one may try to develop its Fourier analysis by direct generalization of classical theory. Such an approach is indeed viable. However, in the process of making the proofs rigorous, the author discovered an independent and simpler proof of the Fourier inversion theorem, which takes advantage of two properties that are due to perfect grading. As is discussed in more detail in Sect. 6, these are: (i) the appearance of a boundary term in the formula for changing to polar integration variables, and (ii) the scale invariance of the Plancherel measure $d \mu(\lambda)$ at infinity.

To complete this introduction, we state the result that is obtained by using the hyperbolic superplane as a toy model for $\langle c\rangle$, see Eq. (5.3):

$$
\langle c\rangle=2 \int_{0}^{\infty} e^{-\left(\lambda^{2}+1 / 4\right) L / \xi} \lambda \tanh (\pi \lambda) d \lambda
$$

An easy computation shows that $\langle c\rangle \cong \xi / L$ for $L \ll \xi$ and

$$
\langle c\rangle \cong 2^{-1} \pi^{3 / 2}(L / \xi)^{-3 / 2} \exp \left(-\frac{L}{4 \xi}\right)
$$

${ }^{1}$ In the present paper the word "graded" means " $Z_{2}$-graded" 
for $L \gg \xi$. The first result is Ohm's law for quasi-one-dimensional systems, and the second corresponds to exponential localization. The corrections to Ohmic behavior ("weak localization") are similar to those for Efetov's model I, describing disordered one-electron systems with potential scattering only (orthogonal universality class).

The material of the present paper is organized as follows. In Sect. 1, the hyperbolic superplane is introduced as a quotient space $\mathbf{G} / \mathbf{K}, \mathbf{G}$ being a connected noncompact semisimple Lie group and $\mathbf{K}$ a maximal compact subgroup. Section 2 contains Theorem 1, which states the correct formula for transforming to the integration variables suggested by the Cartan decomposition for $\mathbf{G}$. The spherical functions for the hyperbolic superplane are introduced, and some of their properties are established, in Sect. 3. The heart of the paper is Sect. 4, where the invertibility of the Fourier transform on $\mathbf{G} / \mathbf{K}$ is proved. The Fourier inversion theorem is then used in Sect. 5 to calculate the average conductance of an ensemble of disordered wires. A discussion is given in Sect. 6; some readers might benefit from reading this section prior to the body of the paper.

\section{Hyperbolic Superplane}

The notation used in the present paper is what the author considers a reasonable synthesis of Berezin's notations in the context of superanalysis [3] and the notation used in Helgason's latest volume on classical geometric analysis [7]. Since Berezin's notational conventions are perhaps not too familiar, a glossary is given in the Appendix.

We introduce the hyperbolic superplane and discuss various geometric concepts associated with it. The construction uses two matrices $\theta$ and $\tau \in \operatorname{GMat}(3,2 \mid \Lambda)$,

$$
\theta=\operatorname{diag}\left(1,-1_{4}\right), \quad \tau=\operatorname{diag}\left(1_{3},\left(\begin{array}{cc}
0 & -1 \\
1 & 0
\end{array}\right)\right),
$$

having the properties $\theta^{2}=1_{5}$ and $\tau^{2}=\mathscr{A}$. Given these, we define a connected noncompact semisimple Lie group $\mathbf{G} \subset \operatorname{GMat}(3,2 \mid \Lambda)$ by

$$
\mathbf{G}=\left\{g \in \mathbf{G M a t}(3,2 \mid \Lambda) \mid \hat{\imath} \bar{g}=g, g^{\dagger}=(\hat{\theta} g)^{-1}, \operatorname{sdet}(g)=1, m\left(g_{00}\right) \geqq 1\right\},
$$

where $\hat{\tau} g=\tau g \tau^{-1} ; \hat{\theta} g=\theta g \theta^{-1} ; g_{a b}$ with $a, b$ taking the values $0,1,2,3,4$ are the elements of the supermatrix $g$; and multiplication is the usual matrix multiplication in $\mathbf{G M a t}(3,2 \mid \Lambda)$. Let $\mathbf{K}$ be the subgroup of $\hat{\theta}$-stable elements of $\mathbf{G}$ :

$$
\mathbf{K}=\{k \in \mathbf{G} \mid \hat{\theta} k=k\} .
$$

Then by the term "hyperbolic superplane" we will mean the quotient space $\mathbf{G} / \mathbf{K}$ consisting of the left cosets $g \mathbf{K}(g \in \mathbf{G}) . e$ will denote the unit element of $\mathbf{G}$, and $o=e \mathbf{K}$ the origin of $\mathbf{G} / \mathbf{K}$.

Taking $\mathbf{G}$ to act on $\mathbf{G} / \mathbf{K}$ by left translation in the usual manner, we endow $\mathbf{G} / \mathbf{K}$ with its natural $\mathbf{G}$-invariant geometry, given by the unique (within normalization) G-invariant metric tensor, $d s^{2}$. We anticipate that in this geometry $\mathbf{G} / \mathbf{K}$ is proper Riemannian in the sense that $m\left(d s^{2}(u, u)\right) \geqq 0$ for any vector field $u$.

There exist various equivalent ways of representing $\mathbf{G} / \mathbf{K}$, of which we mention two. 
(1) $\mathbf{G} / \mathbf{K}$ as a submanifold of $\mathbf{G}$. If $\Pi$ maps $g \in \mathbf{G}$ onto $\Pi(g) \stackrel{\text { def }}{=} g(\hat{\theta} g)^{-1}=g g^{\dagger}$, then $\Pi(\mathbf{G})$ is a submanifold of $\mathbf{G}$ and may be identified with $\mathbf{G} / \mathbf{K}$.

(2) $\mathbf{G} / \mathbf{K}$ as a super Poincaré disk. Consider the (nonlinear) space of supervectors $v \in \mathbb{C}^{2,2}(\Lambda)$ whose components $v_{a}(a=1,2,3,4)$ satisfy the reality constraints $\bar{v}_{1}=v_{1}$, $\bar{v}_{2}=v_{2}, \bar{v}_{3}=v_{4}, \bar{v}_{4}=-v_{3}$ and the condition $m\left(v_{1}^{2}+v_{2}^{2}\right)<1$. Turn this space into a Riemannian supermanifold, $\mathbf{D}$, by endowing it with the metric tensor

$$
\left(1-v^{\dagger} v\right)^{-1} \sum_{a, b=1}^{4} d \bar{v}_{a}\left(1-v v^{\dagger}\right)^{-1}{ }_{a b} d v_{b}
$$

(The dyadic product $v v^{\dagger} \in \operatorname{Mat}(2,2 \mid \Lambda)$ has matrix elements $\left(v v^{\dagger}\right)_{a b}=v_{a} \bar{v}_{b}$, and we define $v^{\dagger} v=\sum_{a} \bar{v}_{a} v_{a}$.) $\mathbf{D}$ is called the Poincaré model of the hyperbolic superplane and is mapped diffeomorphically onto $\mathbf{G} / \mathbf{K}$ by $T_{D}: \mathbf{D} \rightarrow \mathbf{G} / \mathbf{K}$,

$$
v \mapsto T_{D}(v)=\left(\begin{array}{cc}
\left(1-v^{\dagger} v\right)^{-1 / 2} & v^{\dagger}\left(1-v v^{\dagger}\right)^{-1 / 2} \\
v\left(1-v^{\dagger} v\right)^{-1 / 2} & \left(1-v v^{\dagger}\right)^{-1 / 2}
\end{array}\right) \mathbf{K},
$$

the expression (1.1) being the pullback of the $\mathbf{G}$-invariant metric on $\mathbf{G} / \mathbf{K}$ by $T_{D}$. It is evident from (1.2) in combination with the definition of $\mathbf{D}$ that $\mathbf{G} / \mathbf{K}$ is perfectly graded: $\operatorname{dim}(\mathbf{G} / \mathbf{K})=\operatorname{dim}(\mathbf{D})=(2,2)$.

Next, we introduce the Lie algebra of $\mathbf{G}$ :

$$
\mathbf{g}=\left\{X \in \operatorname{Mat}(3,2 \mid \Lambda) \mid \hat{\imath} \bar{X}=X, X^{\dagger}=-\hat{\theta} X\right\}
$$

(g is a "Lie algebra with Grassmann structure" in the terminology of Berezin [3].) It is instructive to contemplate the explicit form of an element $X \in \mathbf{g}$ :

$$
X=\left(\begin{array}{ccccc}
0 & t & b & \bar{\chi} & -\chi \\
t & 0 & -\varphi & -\bar{\eta} & \eta \\
b & \varphi & 0 & -\bar{\kappa} & \kappa \\
\chi & \eta & \kappa & i d & -\bar{z} \\
\bar{\chi} & \bar{\eta} & \bar{\kappa} & z & -i d
\end{array}\right) .
$$

By the definition of $\mathrm{g}$, the variables $t, b, \varphi$ and $d$ are real elements of ${ }^{0} \Lambda, z \in^{0} \Lambda$ is complex, and $\chi, \bar{\chi}, \eta, \bar{\eta}, \kappa$, and $\bar{\kappa}$ are element of ${ }^{1} \Lambda$. With $\operatorname{Ad}(g) X=g X g^{-1}$ being the adjoint action of $\mathbf{G}$ on $\mathbf{g}$, we introduce an $\operatorname{Ad}(\mathbf{G})$-invariant symmetric form $\langle\cdot, \cdot\rangle$ on $\mathbf{g}$ by $\langle X, Y\rangle=\frac{1}{2} \operatorname{str} X Y$. Also, let $\mathbf{g}=\mathbf{k}+\mathbf{p}$ be the Cartan decomposition fixed by the Cartan involution $\hat{\theta}$. Then it follows on general grounds, and it is also evident from (1.3), that $\langle X, X\rangle$ is positive on $m(\mathbf{p})$. Since the restriction of $\langle\cdot, \cdot\rangle$ to $\mathbf{p}$ induces the $\mathbf{G}$-invariant metric on $\mathbf{G} / \mathbf{K}$ via the exponential mapping, $\mathbf{G} / \mathbf{K}$ is proper Riemannian as stated earlier.

An important concept in the analysis on $\mathbf{G} / \mathbf{K}$ is that of a maximal abelian subgroup $\mathbf{A} \subset \boldsymbol{\Pi}(\mathbf{G}) \subset \mathbf{G}$. In the present case $\mathbf{A}$ has dimension one and, to be definite, we take it to be the group of matrices

$$
a_{t}=\operatorname{diag}\left(\left(\begin{array}{cc}
\cosh t & \sinh t \\
\sinh t & \cosh t
\end{array}\right), 1_{3}\right), \quad t \in^{0} \Lambda, t \text { real. }
$$

$\mathbf{A}^{+}$is defined as the subset of elements $a_{t} \in \mathbf{A}$ with $m(t)>0$. The Lie algebra a of 
A consists of the multiples $t H_{0}$, with $t \in^{0} \Lambda$ real, of the generator

$$
H_{0}=\operatorname{diag}\left(\left(\begin{array}{ll}
0 & 1 \\
1 & 0
\end{array}\right), 0_{3}\right) \text {. }
$$

Roots and root vectors of the pair $(\mathbf{a}, \mathbf{g})$ are defined by the equation $\operatorname{ad}\left(t H_{0}\right) X_{\alpha}=\alpha(t) X_{\alpha}$. There exists a single nonvanishing positive root $\alpha(t)=t$, the general element of the corresponding root space being

$$
\left(\begin{array}{ccccc}
0 & 0 & b & \bar{\chi} & -\chi \\
0 & 0 & b & \bar{\chi} & -\chi \\
b & -b & 0 & 0 & 0 \\
\chi & -\chi & 0 & 0 & 0 \\
\bar{\chi} & -\bar{\chi} & 0 & 0 & 0
\end{array}\right) .
$$

Here, $b \in^{0} \Lambda, b$ real, and $\chi, \bar{\chi} \in{ }^{1} \Lambda$. It is useful to assign to each root a multiplicity $m_{\alpha}$. In the case of Lie algebras with Grassmann structure, we define $m_{\alpha}$ as the difference of the (real) dimensions of the even and odd subspaces of the root space corresponding to $\alpha$. Thus, the multiplicity is $1-2=-1$ for the above root. The linear space of elements of the form (1.4) is a nilpotent Lie algebra, denoted by $\mathbf{n}$. The nilpotent subgroup of $\mathbf{G}$ that has $\mathbf{n}$ for its Lie algebra is denoted by $\mathbf{N}$.

\section{Integral Formula for the Cartan Decomposition}

In this section, and at the beginning of the next one, we present, largely without proof, a number of results that will be used in developing the Fourier transform on $\mathbf{G} / \mathbf{K}$ in Sect. 4. Some of them have been proved by Berezin [3], others, since $\mathbf{G}$ is a connected noncompact semisimple Lie group (albeit with Grassmann structure) and $\mathbf{K}$ a maximal compact subgroup, follow rather directly by generalization from classical geometric analysis [7]. However, one especially important result, Theorem 1, has no counterpart in classical analysis, and in this case a detailed proof will be given.

Let us agree that the integration domain for the commuting integration variables in any Berezin integral written $\int_{\mathbf{P}} \ldots$ is $m(\mathbf{P})$, to keep the notation simple. Let us further agree that for a supermanifold $\mathbf{P}$ of dimension $(p, q)$, the term "function on P" abbreviates " $\Lambda_{q}$-valued function on the $p$-dimensional base manifold of $\mathbf{P}$." With this terminology, we denote by $\mathscr{D}(\mathbf{G} / \mathbf{K})$ the space of compactly supported $C^{\infty}$-functions on $\mathbf{G} / \mathbf{K}$. There exists a $\mathbf{G}$-invariant Berezin integral

$$
\int_{\mathbf{G} / \mathbf{K}} D g_{\mathbf{K}} f\left(g_{0} g \cdot o\right)=\int_{\mathbf{G} / \mathbf{K}} D g_{\mathbf{K}} f(g \cdot o)
$$

which is well-defined and has a coordinate-independent meaning for $f \in \mathscr{D}(\mathbf{G} / \mathbf{K})$ [3]. A natural normalization is provided by the proof of Theorem 1 below.

Given the Iwasawa decomposition $\mathbf{G}=\mathbf{N A K}[7]$ in the form

$$
g=n(g) a(g) k(g), \quad a(g)=\exp \left(t(g) H_{0}\right),
$$

the relations

$$
k(m g)=m k(g), \quad t(m g)=t(g)
$$


are true if $m$ is an element of $\mathbf{M}$, the centralizer of $\mathbf{A}$ in $\mathbf{K}$. Let $\rho$ be half the sum of a set of positive roots,

$$
\rho(t)=\frac{1}{2} \sum_{\alpha>0} m_{\alpha} \alpha(t)=-t / 2 .
$$

It is stated in Proposition 3.8 in Chap. II of ref. [7] that, when $\mathbf{N}$ acts on $\mathbf{G} / \mathbf{K}$, the radial part of $\Delta_{\mathbf{G} / \mathbf{K}}$ for the transversal submanifold $\mathbf{A} \cdot \boldsymbol{o}$ is given by

$$
e^{\rho} \Delta_{\mathrm{A}} e^{-\rho}-|\rho|^{2},
$$

where $\Delta_{\mathrm{A}}$ is the (Euclidean) Laplacian on $\mathbf{A} \cdot o, e^{\rho}$ denotes the function $a \cdot o \mapsto e^{\rho(t(a))}$ on $\mathbf{A} \cdot o$ and, in the present case, $|\rho|^{2}=1 / 4$.

Our next subject is an integral formula for functions on $\mathbf{G} / \mathbf{K}$ which is related to the Cartan decomposition $\mathbf{G}=\mathbf{K A}^{+} \mathbf{K}$ [7]. Let $d a$ denote the Euclidean measure on $\mathbf{A}$. (Measures written with a capital $D$ are genuine Berezin measures, whereas $d a$ is an ordinary measure not involving any anticommuting variables.) The mapping $(k \mathbf{M}, a) \mapsto k a \mathbf{K}$ is a diffeomorphism of $(\mathbf{K} / \mathbf{M}) \times \mathbf{A}^{+}$onto the set of regular elements of $\mathbf{G} / \mathbf{K}$. Therefore, denoting by $D k_{\mathbf{M}}$ the $\mathbf{K}$-invariant Berezin measure on $\mathbf{K} / \mathbf{M}$, and introducing

$$
\delta\left(\exp r H_{0}\right) \stackrel{\text { def }}{=} \prod_{\alpha>0}(\sinh \alpha(r))^{m_{\alpha}}=(\sinh r)^{-1},
$$

we might expect that $\int_{\mathbf{G} / \mathbf{K}} D g_{\mathbf{K}} f(g \cdot o)$ equals

$$
\int_{\mathbf{A}^{+}}\left(\int_{\mathbf{K} / \mathbf{M}} D k_{\mathbf{M}} f(k a \cdot o)\right) \delta(a) d a,
$$

by the analogy with classical geometric analysis; see Theorem 5.8 in Chap. I of ref. [7]. However, it is the following, modified statement that is true. Let the normalization of the measures $D g_{\mathbf{K}}, D k_{\mathbf{M}}$ and $d a$ be fixed by Eq. (2.7) and the text preceding it.

Theorem 1. For any $f \in \mathscr{D}(\mathbf{G} / \mathbf{K})$,

$$
\int_{\mathbf{G} / \mathbf{K}} D g_{\mathbf{K}} f(g \cdot o)=f(o)+\int_{\mathbf{A}^{+}}\left(\int_{\mathbf{K} / \mathbf{M}} D k_{\mathbf{M}} f(k a \cdot o)\right) \delta(a) d a .
$$

Proof. We will prove the theorem by explicitly writing out both sides in suitable coordinates and carefully performing the relevant variable changes. Consider the map

$$
(r, \varphi, \bar{\eta}, \eta) \mapsto T_{C}(r, \varphi, \bar{\eta}, \eta)=\exp \left(\begin{array}{ccccc}
0 & 0 & 0 & 0 & 0 \\
0 & 0 & -\varphi & 0 & 0 \\
0 & \varphi & 0 & 0 & 0 \\
0 & 0 & 0 & 0 & 0 \\
0 & 0 & 0 & 0 & 0
\end{array}\right)
$$




$$
\exp \left(\begin{array}{ccccc}
0 & 0 & 0 & 0 & 0 \\
0 & 0 & 0 & -\bar{\eta} & \eta \\
0 & 0 & 0 & 0 & 0 \\
0 & \eta & 0 & 0 & 0 \\
0 & \bar{\eta} & 0 & 0 & 0
\end{array}\right) \exp \left(r H_{0}\right) \mathbf{K}
$$

Here, $r$ subject to $m(r)>0$ parametrizes $\mathbf{A}^{+}$by $r \mapsto \exp r H_{0}$, while $\varphi, \bar{\eta}$ and $\eta$ serve as coordinates of $\mathbf{K} / \mathbf{M}$. By evaluating the square root of the superdeterminant of the $\mathbf{G}$-invariant metric tensor, the components of which are given by

$$
(d r)^{2}+\left(\sinh ^{2} r\right)(1-2 \bar{\eta} \eta)(d \varphi)^{2}+2\left(\sinh ^{2} r\right)(1-\bar{\eta} \eta) d \bar{\eta} d \eta
$$

in these coordinates, we find $T_{C}^{*}\left(D g_{\mathbf{K}}\right)=$ const $\times(\sinh r)^{-1} d r d \varphi \partial_{\bar{\eta}} \partial_{\eta}$, where $\partial_{\eta}=\partial / \partial \eta$. Here, and throughout the paper, we fix the normalization of $D g_{\mathbf{K}}$ by taking the constant to be $(2 \pi)^{-1}$. Normalizing the measure $D k_{\mathbf{M}} \delta(a) d a$ accordingly, we have the result

$$
\int_{\mathbf{A}^{+}}\left(\int_{\mathbf{K} / \mathbf{M}} D k_{\mathbf{M}} f(k a \cdot o)\right) \delta(a) d a=\frac{1}{2 \pi} \int_{0}^{\infty}\left(\int_{0}^{2 \pi} \partial_{\bar{\eta}} \partial_{\eta} f\left(T_{C}(r, \varphi, \bar{\eta}, \eta)\right) d \varphi\right)(\sinh r)^{-1} d r .
$$

On the left-hand side of (2.4) we use the coordinates (1.2), writing

$$
v(x, y, \bar{\zeta}, \zeta)=\left(\begin{array}{l}
x \\
y \\
\zeta \\
\bar{\zeta}
\end{array}\right)
$$

The image of $D g_{\mathbf{K}}$ under the inverse of $T_{D}$ is given by

$$
T_{D}^{*}\left(D g_{\mathbf{K}}\right)=D v\left(1-v^{\dagger} v\right)^{-1 / 2}, \quad \text { where } \quad D v=(2 \pi)^{-1} d x d y \partial_{\bar{\zeta}} \partial_{\zeta}
$$

as follows from the expression for the G-invariant metric on D, Eq. (1.1). Setting

$$
F_{0}(x, y, \bar{\zeta}, \zeta)=\left(1-x^{2}-y^{2}-2 \overline{\zeta \zeta}\right)^{-1 / 2} f\left(T_{D}(v(x, y, \bar{\zeta}, \zeta))\right)
$$

we obtain

$$
\int_{\mathbf{G} / \mathbf{K}} D g_{\mathbf{K}} f(g \cdot o)=\frac{1}{2 \pi} \int_{x^{2}+y^{2}<1} \partial_{\bar{\zeta}} \partial_{\zeta} F_{0}(x, y, \bar{\zeta}, \zeta) d x d y
$$

By comparing (1.2) with (2.5), we see that the coordinates $(x, y, \bar{\zeta}, \zeta)$ and $(r, \varphi, \bar{\eta}, \eta)$ are related in the following way:

$$
\left(\begin{array}{l}
x \\
y \\
\bar{\zeta} \\
\zeta
\end{array}\right)=\tanh r\left(\begin{array}{c}
\cos \varphi \sqrt{1-2 \bar{\eta} \eta} \\
\sin \varphi \sqrt{1-2 \bar{\eta} \eta} \\
\bar{\eta} \\
\eta
\end{array}\right) .
$$

We will now change integration variables in three steps. First, we introduce polar coordinates according to $x=u \cos \varphi, y=u \sin \varphi, \bar{\zeta}=u \bar{\eta}$, and $\zeta=u \eta$, which leads to

$$
\int_{\mathbf{G} / \mathbf{K}} D g_{\mathbf{K}} f(g \cdot o)=\frac{1}{2 \pi} \int_{0}^{1}\left(\int_{0}^{2 \pi} \partial_{\bar{\eta}} \partial_{\eta} F_{1}(u, \varphi, \bar{\eta}, \eta) d \varphi\right) u^{-1} d u,
$$


where $F_{1}(u, \varphi, \bar{\eta}, \eta)=F_{0}(u \cos \varphi, u \sin \varphi, u \bar{\eta}, u \eta)$. In the second step, we set

$$
u=w \sqrt{1-2 \bar{\eta} \eta}=w-\bar{\eta} \eta w
$$

all other variables remaining unchanged. It is known from [3], and has been further elaborated upon in [10], that a variable transformation involving the shift of an even variable by nilpotent terms may give rise to boundary contributions. The extra term $f(o)$ in (2.4) is precisely such a boundary term, being generated by the change of variable (2.10). To see how this comes about, consider

$$
\begin{aligned}
& \frac{1}{2 \pi} \int_{0}^{1}\left(\int_{0}^{2 \pi} \partial_{\bar{\eta}} \partial_{\eta} F_{1}(w-\bar{\eta} \eta w, \varphi, \bar{\eta}, \eta) d \varphi\right) w^{-1} d w \\
& \quad=\frac{1}{2 \pi} \int_{0}^{1}\left(\int_{0}^{2 \pi} \partial_{\bar{\eta}} \partial_{\eta}\left(F_{1}(u, \varphi, \bar{\eta}, \eta)-\bar{\eta} \eta u \frac{\partial}{\partial u} F_{1}(u, \varphi, 0,0)\right) d \varphi\right) u^{-1} d u
\end{aligned}
$$

where we have expanded $F_{1}$ with respect to the nilpotent quantity in its first argument. The total derivative term can be integrated and yields, since the contribution from the upper boundary vanishes by the assumption of compact support for $f$, the result $-F_{1}(0, \cdot, \cdot, \cdot)=-F_{0}(0,0,0,0)=-f(o)$. Hence,

$$
\int_{\mathbf{G} / \mathbf{K}} D g_{\mathbf{K}} f(g \cdot o)=f(o)+\frac{1}{2 \pi} \int_{0}^{1}\left(\int_{0}^{2 \pi} \partial_{\bar{\eta}} \partial_{\eta} F_{2}(w, \varphi, \bar{\eta}, \eta) d \varphi\right) w^{-1} d w
$$

where $F_{2}(w, \varphi, \bar{\eta}, \eta)=F_{1}(w \sqrt{1-2 \bar{\eta} \eta}, \varphi, \bar{\eta}, \eta)$. In the final step, we recall Eq. (2.8) and set $w=\tanh r, F_{3}(r, \varphi, \bar{\eta}, \eta)=(\cosh r)^{-1} F_{2}(\tanh r, \varphi, \bar{\eta}, \eta)$, thereby turning Eq. (2.11) into

$$
\int_{\mathbf{G} / \mathbf{K}} D g_{\mathbf{K}} f(g \cdot o)=f(o)+\frac{1}{2 \pi} \int_{0}^{\infty}\left(\int_{0}^{2 \pi} \partial_{\bar{\eta}} \partial_{\eta} F_{3}(r, \varphi, \bar{\eta}, \eta) d \varphi\right)(\sinh r)^{-1} d r .
$$

This last integral is nothing but the second term on the right-hand side of Eq. (2.4) since $F_{3}=f \circ T_{C}$, as is seen by recalling Eqs. (2.7) and (2.9) and forming the composition of the three variable transformations given.

Corollary. It is evident from the above proof that

$$
\int_{\mathbf{K} / \mathbf{M}} D k_{\mathbf{M}}=(2 \pi)^{-1} \int_{0}^{2 \pi}\left(\partial_{\bar{\eta}} \partial_{\eta} \cdot 1\right) d \varphi=0
$$

\section{Spherical Functions}

A central role in the Fourier analysis on $\mathbf{G} / \mathbf{K}$ is played by the so-called spherical functions on $\mathbf{G} / \mathbf{K}$, introduced in the present section.

Taking $t(g)$ to be the coordinate appearing in the Iwasawa decomposition (2.1), we define for $\lambda \in \mathbb{C}$ functions $\phi_{\lambda}$ on $\mathbf{G}$ by (Harish-Chandra)

$$
\phi_{\lambda}(g)=\int_{\mathbf{K} / \mathbf{M}} D k_{\mathbf{M}} \exp \left((-1 / 2+i \lambda) t\left(k^{-1} g\right)\right) .
$$

This definition makes sense since $t(\mathbf{M} g)=t(g)$, see relations (2.2). Clearly, $\phi_{\lambda}$ is bi-invariant under $\mathbf{K}$, which is to say that $\phi_{\lambda}$ satisfies $\phi_{\lambda}\left(k_{1} g k_{2}\right)=\phi_{\lambda}(g)$ for $k_{1}, k_{2} \in \mathbf{K}$. 
We may therefore regard $\phi_{\lambda}$ as a $\mathbf{K}$-radial function on $\mathbf{G} / \mathbf{K}$ (i.e. as a function on $\mathbf{G} / \mathbf{K}$ left-invariant under $\mathbf{K}$ ) and, since the Laplace-Beltrami operator $\Delta_{\mathbf{G} / \mathbf{K}}$ commutes with left translations and has the radial part given in (2.3), $\phi_{\lambda}$ is an eigenfunction of $\Delta_{\mathbf{G} / \mathbf{K}}$, the eigenvalue being $-\left(\lambda^{2}+\frac{1}{4}\right)$. We will call the functions $\phi_{\lambda}$ the spherical functions on $\mathbf{G} / \mathbf{K}$, by a natural extension of classical terminology [7]. Notice one important feature: $\phi_{\lambda}$ vanishes at the origin since, by Eqs. (3.1) and (2.12),

$$
\phi_{\lambda}(e)=\int_{\mathbf{K} / \mathbf{M}} D k_{\mathbf{M}}=0 .
$$

This means that one cannot fix the normalization of the spherical functions by requiring $\phi_{\lambda}(e)=1$ as in the classical case. A natural normalization is that given by Eq. (3.1), with $D k_{\mathbf{M}}$ normalized as implied by the first equality in (2.12).

In the proof of the Fourier inversion theorem we will use the identity (Lemma 4.4 in Chap. IV of ref. [7])

$$
\phi_{\lambda}\left(g^{-1} h\right)=\int_{\mathbf{K} / \mathbf{M}} D k_{\mathbf{M}} e^{(-1 / 2-i \lambda) t\left(k^{-1} g\right)} e^{(-1 / 2+i \lambda) t\left(k^{-1} h\right)} .
$$

Setting in this relation $h=e$, we find $\phi_{\lambda}\left(g^{-1}\right)=\phi_{-\lambda}(g)$, and, because $a^{-1}=\hat{\theta} a=\theta a \theta^{-1}$ and $\theta \in \mathbf{K}$,

$$
\phi_{\lambda}(a)=\phi_{-\lambda}\left(a^{-1}\right)=\phi_{-\lambda}(a)=\phi_{\lambda}\left(a^{-1}\right) .
$$

The study of the behavior of $\phi_{\lambda}$ for large values of $r$ leads to the introduction of Harish-Chandra's c-function, defined for $\operatorname{Re}(i \lambda)>0$ by

$$
\mathbf{c}(\lambda)=\lim _{r \rightarrow+\infty} e^{(-1 / 2-i \lambda) r} \phi_{\lambda}\left(\exp r H_{0}\right) .
$$

The most economical way of evaluating $\mathbf{c}(\lambda)$ is provided by Theorem 6.14 in Chap. IV of ref. [7], stating that

$$
\mathbf{c}(\lambda)=\int_{\overline{\mathbf{N}}} D \bar{n} e^{(-1 / 2+i \lambda) t(\bar{n})}
$$

where $D \bar{n}$ is the (suitably normalized) invariant Berezin measure of the rilpotent group $\overline{\mathbf{N}}=\hat{\theta} \mathbf{N}$. Parametrizing $\overline{\mathbf{N}}$ through its Lie algebra, and using Theorem 3.8, Chap. IX, and Corollary 4.4, Chap. VI, of ref. [6], we obtain for $\mathbf{c}(\lambda)$ the integral

$$
\mathbf{c}(\lambda)=\frac{1}{4 \pi} \int_{\mathbb{R}}\left(\partial_{\bar{\chi}} \partial_{\chi}\left(1+b^{2}+2 \bar{\chi} \chi\right)^{1 / 2-i \lambda}\right) d b,
$$

which in fact converges for $\operatorname{Re}(i \lambda)>0$. Evaluation gives

$$
\mathbf{c}(\lambda)=\frac{1}{2 \sqrt{\pi}} \frac{\Gamma(i \lambda)}{\Gamma\left(-\frac{1}{2}+i \lambda\right)} .
$$

This result for $\mathbf{c}(\lambda)$ can be extended to a meromorphic function on the entire complex $\lambda$-plane. For real $\lambda$ in particular,

$$
|\mathbf{c}(\lambda)|^{-2}=(\mathbf{c}(\lambda) \mathbf{c}(-\lambda))^{-1}=4 \pi \frac{\lambda \tanh (\pi \lambda)}{\lambda^{2}+\frac{1}{4}},
$$

by standard identities for the Gamma-function [1]. 
We now derive the series expansion for $\phi_{\lambda}$ in terms of exponential functions. From expression (2.6) for the $\mathbf{G}$-invariant metric, we know that the $\mathbf{K}$-radial part of the Laplace-Beltrami operator $\Delta_{\mathbf{G} / \mathbf{K}}$ is given by $(\sinh r) \partial_{r}(\sinh r)^{-1} \partial_{r}$. Writing it in the form $\left(\partial_{r} \partial_{r}-\operatorname{coth} r \partial_{r}\right)$, we see that $\phi_{\lambda}\left(\exp r H_{0}\right)$ satisfies the differential equation

$$
\left(\partial_{r} \partial_{r}-\operatorname{coth} r \partial_{r}+\left(\lambda^{2}+1 / 4\right)\right) F(r)=0 .
$$

In the limit $r \rightarrow+\infty$ this equation contracts to $\left(\partial_{r} \partial_{r}-\partial_{r}+\left(\lambda^{2}+1 / 4\right)\right) F(r)=0$, which is solved by the functions $e^{(1 / 2 \pm i \lambda)}$. The hyperbolic cotangent has the series expansion

$$
\operatorname{coth} r=1+2 \sum_{n=1}^{\infty} e^{-2 n r}
$$

which converges for $r>0$. This leads us to seek a solution of Eq. (3.7) in the form

$$
F(r)=\mathrm{e}^{(1 / 2+i \lambda) r} \sum_{n=0}^{\infty} \gamma_{n}(\lambda) e^{-2 n r},
$$

where we take $\gamma_{0}(\lambda)=1$. Inserting (3.8) into (3.7), and comparing the coefficients of the functions $e^{(1 / 2+i \lambda-2 n) r}$ for $n=1,2, \ldots$, we obtain the recursion relation

$$
\gamma_{n}(\lambda)=-(n(n-i \lambda))^{-1} \sum_{l=1}^{n}(n-l-1 / 4-i \lambda / 2) \gamma_{n-l}(\lambda)
$$

which defines $\gamma_{n}(\lambda)$ as a rational function of $\lambda$. To decide whether (3.8) with $\gamma_{n}(\lambda)$ determined by (3.9) in fact provides a solution of (3.7) for $r>0$, we must investigate the convergence properties of the sum over $n$. Although this sum can be shown to converge for all complex $i \lambda \notin \mathbb{N}$, it will suffice for our purposes to prove a weaker statement.

Lemma 1. $\left|\gamma_{n}(\lambda)\right| \leqq 1$ for complex $\lambda$ with $\operatorname{Re}(i \lambda) \leqq 1 / 2$.

Proof. Taking the absolute value on both sides of (3.9), we get the inequality

$$
\left|\gamma_{n}(\lambda)\right| \leqq \sum_{l=1}^{n} \frac{\left|n-l-\frac{1}{4}-\frac{1}{2} i \lambda\right|}{|n(n-i \lambda)|}\left|\gamma_{n-l}(\lambda)\right|
$$

Let us set $\lambda=-\frac{i}{2}+a+i b$ with $a, b$ real and $b \geqq 0$. Then

$$
\frac{\left|n-l-\frac{1}{4}-\frac{1}{2} i \lambda\right|}{|n(n-i \lambda)|}=\frac{1}{n}\left(\frac{\left(n-\frac{1}{2}-l+\frac{1}{2} b\right)^{2}+\frac{1}{2} a^{2}}{\left(n-\frac{1}{2}+b\right)^{2}+a^{2}}\right)^{1 / 2} \leqq \frac{1}{n} .
$$

The inequality $\left|\gamma_{n}(\lambda)\right| \leqq \frac{1}{n} \sum_{l=1}^{n}\left|\gamma_{n-l}(\lambda)\right|$ thus holds for $\operatorname{Re}(i \lambda) \leqq \frac{1}{2}$, and the lemma follows by induction on $n$.

Lemma 2. Let $\gamma_{0}(\lambda)=1$ and define $\gamma_{n}(\lambda)(n=1,2, \ldots)$ recursively through Eq. (3.9). Then (i) the function $\Phi_{\lambda}(r)$ defined for $r>0$ by

$$
\Phi_{\lambda}(r)=\sum_{n=0}^{\infty} \gamma_{n}(\lambda) e^{-2 n r}
$$


is holomorphic in $\lambda$ for $\operatorname{Re}(i \lambda) \leqq 1 / 2$, and (ii)

$$
\phi_{\lambda}\left(\exp r H_{0}\right)=\mathbf{c}(\lambda) e^{(1 / 2+i \lambda) r} \Phi_{\lambda}(r)+\mathbf{c}(-\lambda) e^{(1 / 2-i \lambda) r} \Phi_{-\lambda}(r)
$$

holds for any $r>0$ and $|\operatorname{Re}(i \lambda)| \leqq 1 / 2$.

Proof. It is seen from Eq. (3.9) that the functions $\gamma_{n}(\lambda)$ are holomorphic for $i \lambda \notin \mathbb{N}$. Statement (i) is thus clear since, by Lemma 1, the series for $\Phi_{\lambda}$ converges absolutely and uniformly in $\lambda$ in the designated range. Turning to statement (ii), we first observe that, owing to the absolute convergence of the series for $\Phi_{\lambda}(r)$, the function $F_{+}(r)=e^{(1 / 2+i \lambda) r} \Phi_{\lambda}(r)$ indeed solves Eq. (3.7) for $r>0$ and $\operatorname{Re}(i \lambda) \leqq 1 / 2$. The same holds true for the function $F_{-}(r)=e^{(1 / 2-i \lambda) r} \Phi_{-\lambda}(r)$ for $r>0$ and $\operatorname{Re}(i \lambda) \geqq-1 / 2$. Because they are linearly independent functions of $r, F_{+}$and $F_{-}$constitute a fundamental system of solutions to the second-order ordinary differential equation (3.7). The spherical functions $\phi_{\lambda}$ must therefore be expressible as

$$
\phi_{\lambda}\left(\exp r H_{0}\right)=a_{1}(\lambda) e^{(1 / 2+i \lambda) r} \Phi_{\lambda}(r)+a_{2}(\lambda) e^{(1 / 2-i \lambda) r} \Phi_{-\lambda}(r)
$$

for $r>0$ and $|\operatorname{Re}(i \lambda)| \leqq 1 / 2$. The coefficients $a_{1}(\lambda)$ and $a_{2}(\lambda)$ are determined by the requirement $\phi_{\lambda}=\phi_{-\lambda}$ (Eq. (3.3)) and the asymptotic behavior (3.4)

Remark. The condition on the range of $\operatorname{Re}(i \lambda)$ is imposed for convenience and is more restrictive than is necessary for the validity of the lemma.

\section{The Fourier Transform and Its Inverse}

Mathematical experience [7] suggests that the spherical functions $\phi_{\lambda}$ for $\lambda$ real and positive should in some sense be an orthogonal and complete set of functions. More specifically, we expect that the integral $\int_{\mathbb{R}^{+}} \phi_{\lambda} d \mu(\lambda)$, with $d \mu(\lambda)$ proportional to $|\mathbf{c}(\lambda)|^{-2} d \lambda$ and $|\mathbf{c}(\lambda)|^{-2}$ given by Eq. (3.6), has the properties of a Dirac $\delta$-distribution. We will prove that such is indeed the case. We begin with the following

Lemma 3. Let $d \mu(\lambda)=(4 \pi)^{-1}|\mathbf{c}(\lambda)|^{-2} d \lambda$. Then for all $r>0$,

$$
\lim _{B \rightarrow \infty} \int_{0}^{B} \phi_{\lambda}\left(\exp r H_{0}\right) d \mu(\lambda)=-1 .
$$

Proof. We will make use of the decomposition of $\phi_{\lambda}$ given by Lemma 2. Consider thus the integral

$$
\int_{-B}^{+B} \mathbf{c}(\lambda) e^{(1 / 2+i \lambda) r} \Phi_{\lambda}(r) d \mu(\lambda)=\frac{1}{4 \pi} \int_{-B}^{+B}(\mathbf{c}(-\lambda))^{-1} e^{(1 / 2+i \lambda) r}\left(\sum_{n=0}^{\infty} \gamma_{n}(\lambda) e^{-2 n r}\right) d \lambda .
$$

By Eqs. $(3.5,3.9)$ and Lemma 1, the integrand is continuous and bounded in $\lambda$. We first establish the existence of the limit $B \rightarrow \infty$ by discussing the asymptotic behavior of the integrand. In the limit $\lambda \rightarrow+\infty$ the functions $\gamma_{n}(\lambda)$ tend to definite values given recursively by $\gamma_{0}(\infty)=1, \gamma_{n}(\infty)=-\frac{1}{2 n} \sum_{l=1}^{n} \gamma_{n-l}(\infty)$. Furthermore,

$$
\lim _{\lambda \rightarrow+\infty}(-i \lambda)^{1 / 2}(\mathbf{c}(-\lambda))^{-1}=2 \sqrt{\pi} \lim _{\lambda \rightarrow+\infty}(-i \lambda)^{1 / 2} \frac{\Gamma\left(-\frac{1}{2}-i \lambda\right)}{\Gamma(-i \lambda)}=2 \sqrt{\pi},
$$


by a standard result for the Gamma-function [1]. Similar formulas hold for $\lambda \rightarrow-\infty$. Now, since

$$
\int_{B}^{\infty} \lambda^{-1 / 2} e^{i \lambda r} d \lambda=i B^{-1 / 2} r^{-1} e^{i B r}-\frac{i}{2 r} \int_{B}^{\infty} \lambda^{-3 / 2} e^{i \lambda r} d \lambda
$$

it follows that the expression (4.1) tends to a definite limit as $B \rightarrow \infty$. The integral $\int_{\mathbb{R}}(\mathbf{c}(-\lambda))^{-1} e^{(1 / 2+i \lambda) r} \Phi_{\lambda}(r) d \lambda$ is conveniently evaluated by closing the integration contour around the upper half of the complex $\lambda$-plane and using Cauchy's Theorem. According to Lemma 2, $\Phi_{\lambda}(r)$ is holomorphic in a domain including the upper half-plane, for $r>0$. The singularities of $(\mathbf{c}(-\lambda))^{-1}$ all lie in the lower half-plane, except for a simple pole at $\lambda=i / 2$, see Eq. (3.5). At the point $\lambda=i / 2$ the functions $\gamma_{n}(\lambda)$ all vanish for $n \geqq 1$, as follows immediately from (3.9). Calculating the residue, we easily find $\int_{\mathbb{R}} \mathbf{c}(\lambda) e^{(1 / 2+i \lambda) r} \Phi_{\lambda}(r) d \mu(\lambda)=-1$, and thus, by (3.3), Lemma 2 , and the invariance of $\int_{\mathbb{R}}^{\mathbb{R}} \phi_{\lambda} d \mu(\lambda)$ under $\lambda \mapsto-\lambda$,

$$
\begin{aligned}
\int_{\mathbb{R}^{+}} \phi_{\lambda}\left(\exp r H_{0}\right) d \mu(\lambda) & =\frac{1}{2} \int_{\mathbb{R}} \phi_{\lambda}\left(\exp r H_{0}\right) d \mu(\lambda) \\
& =\int_{\mathbb{R}} \mathbf{c}(\lambda) e^{(1 / 2+i \lambda) r} \Phi_{\lambda}(r) d \mu(\lambda)=-1 .
\end{aligned}
$$

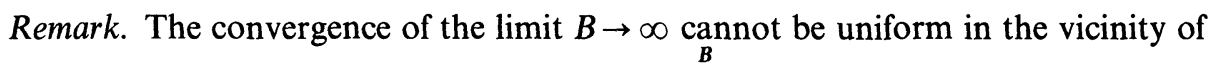
$r=0$ since $\phi_{\lambda}(e)=0$ trivially implies $\lim _{B \rightarrow \infty} \int_{0}^{B} \phi_{\lambda}(e) d \mu(\lambda)=0$. Of course, this non-uniformity is to be expected since a " $\delta$-function singularity" resides at $r=0$.

Next, we define a kernel $\delta_{R}: f \mapsto \delta_{R} f$ acting on functions $f \in \mathscr{D}(\mathbf{G} / \mathbf{K})$ by

$$
\left(\delta_{R} f\right)(h \cdot o)=\lim _{B \rightarrow \infty} \int_{0}^{B}\left(\int_{\mathbf{G} / \mathbf{K}} D g_{\mathbf{K}} \phi_{\lambda}\left(g^{-1} h\right) f(g \cdot o)\right) d \mu(\lambda) .
$$

Let $\mathscr{D}_{0}(\mathbf{G} / \mathbf{K}) \subset \mathscr{D}(\mathbf{G} / \mathbf{K})$ denote the subspace of functions $f$ with vanishing Berezin-integral $\int_{\mathbf{G} / \mathbf{K}} D g_{\mathbf{K}} f(g \cdot o)$.

Lemma 4. $\delta_{R} f=f$ for all $f \in \mathscr{D}_{0}(\mathbf{G} / \mathbf{K})$.

Proof. By using the G-invariance of $D g_{\mathbf{K}}$ and Theorem 1, we get

$$
\begin{aligned}
\int_{\mathbf{G} / \mathbf{K}} D g_{\mathbf{K}} \phi_{\lambda}\left(g^{-1} h\right) f(g \cdot o) & =\int_{\mathbf{G} / \mathbf{K}} D g_{\mathbf{K}} \phi_{\lambda}\left(g^{-1}\right) f(h g \cdot o) \\
& =\int_{\mathbf{A}^{+}}\left(\int_{\mathbf{K} / \mathbf{M}} D k_{\mathbf{M}} f(h k a \cdot o)\right) \phi_{\lambda}\left(a^{-1}\right) \delta(a) d a .
\end{aligned}
$$

Changing integration variables does not generate any extra term here since $\phi_{\lambda}(e)=0$. In the next step, we insert (4.3) into (4.2). Having done this we observe that

$$
\left(\int_{\mathbf{K} / \mathbf{M}} D k_{\mathbf{M}} f\left(h k \exp r H_{0} \cdot o\right)\right) \times \delta\left(\exp r H_{0}\right)
$$

tends to a definite limit as $r \rightarrow 0$ since the first factor vanishes (at least) linearly with $r$, thereby cancelling the simple pole singularity of the second one. Therefore, in spite of the non-uniformity of the limit $B \rightarrow \infty$, we may take $B$ to infinity before 
integrating over $\mathbf{A}^{+}$:

$$
\left(\delta_{R} f\right)(g \cdot o)=\int_{\mathbf{A}^{+}}\left(\lim _{\boldsymbol{B} \rightarrow \infty} \int_{0}^{B} \phi_{\lambda}\left(a^{-1}\right) d \mu(\lambda)\right)\left(\int_{\mathbf{K} / \mathbf{M}} D k_{\mathbf{M}} f(g k a \cdot o)\right) \delta(a) d a .
$$

We now use (3.3) and Lemma 3, and we add and subtract $f(h \cdot o)$ :

$$
\left(\delta_{R} f\right)(h \cdot o)=f(h \cdot o)-\left\{f(h \cdot o)+\int_{\mathbf{A}^{+}}\left(\int_{\mathbf{K} / \mathbf{M}} D k_{\mathbf{M}} f(h k a \cdot o)\right) \delta(a) d a\right\} .
$$

By Theorem 1, the expression in brackets equals $\int_{\mathbf{G} / \mathbf{K}} D g_{\mathbf{K}} f(h g \cdot o)=\int_{\mathbf{G} / \mathbf{K}} D g_{\mathbf{K}} f(g \cdot o)$, which vanishes by assumption. This proves the lemma.

Corollary. When $\int_{\mathbf{G} / \mathbf{K}} D g_{\mathbf{K}} f(g \cdot o) \neq 0, E q$. (4.4) shows that

$$
f=\delta_{R} f+\left(\int_{\mathbf{G} / \mathbf{K}} D g_{\mathbf{K}} f(g \cdot o)\right) \times 1,
$$

where 1 is the constant function with value unity.

With these preparations, we have at hand all the tools needed to formulate the Fourier transform and prove it invertibility. For $f \in \mathscr{D}(\mathbf{G} / \mathbf{K})$ let the Fourier transform $f \mapsto \tilde{f}$ be defined by

$$
\tilde{f}(\lambda, k)=\int_{\mathbf{G} / \mathbf{K}} D g_{\mathbf{K}} e^{(-1 / 2-i \lambda) t\left(k^{-1} g\right)} f(g \cdot o) .
$$

Note that because of $t(\mathbf{M} g)=t(g), \tilde{f}(\lambda, \cdot)$ satisfies $\tilde{f}(\lambda, k \mathbf{M})=\tilde{f}(\lambda, k)$ and is thus a function on $\mathbf{K} / \mathbf{M}$.

Theorem 2. The Fourier transform (4.5) is invertible for $f \in \mathscr{D}_{0}(\mathbf{G} / \mathbf{K})$ and

$$
f(g \cdot o)=\int_{\mathbb{R}^{+}}\left(\int_{\mathbf{K} / \mathbf{M}} D k_{\mathbf{M}} \tilde{f}(\lambda, k) e^{(-1 / 2+i \lambda) t\left(k^{-1} g\right)}\right) d \mu(\lambda) .
$$

Proof. The theorem follows immediately from Lemma 4 in combination with the property (3.2) of the spherical functions $\phi_{\lambda}$ :

$$
\begin{aligned}
f(h \cdot o) & =\left(\delta_{R} f\right)(h \cdot o)=\lim _{\mathbf{B} \rightarrow \infty} \int_{0}^{B}\left(\int_{\mathbf{G} / \mathbf{K}} D g_{\mathbf{K}} \phi_{\lambda}\left(g^{-1} h\right) f(g \cdot o)\right) d \mu(\lambda) \\
& =\int_{\mathbb{R}^{+}}\left(\int_{\mathbf{K} / \mathbf{M}} D k_{\mathbf{M}} e^{(-1 / 2+i \lambda) t\left(k^{-1} h\right)}\right)\left(\int_{\mathbf{G} / \mathbf{K}} D g_{\mathbf{K}} e^{(-1 / 2-i \lambda) t\left(k^{-1} g\right)} f(g \cdot o)\right) d \mu(\lambda) \\
& =\int_{\mathbb{R}^{+}}\left(\int_{\mathbf{K} / \mathbf{M}} D k_{\mathbf{M}} \tilde{f}(\lambda, k) e^{(-1 / 2+i \lambda) t\left(k^{-1} h\right)}\right) d \mu(\lambda) .
\end{aligned}
$$

Corollary. By the corollary to Lemma 4, the theorem extends to

$$
f(h \cdot o)=\int_{\mathbf{G} / \mathbf{K}} D g_{\mathbf{K}} f(h g \cdot o)+\int_{\mathbb{R}^{+}}\left(\int_{\mathbf{K} / \mathbf{M}} D k_{\mathbf{M}} \tilde{f}(\lambda, k) e^{(-1 / 2+i \lambda) t\left(k^{-1} h\right)}\right) d \mu(\lambda)
$$

for $f \in \mathscr{D}(\mathbf{G} / \mathbf{K})$. 


\section{Application to Disordered Wires}

As was stated in the introduction, the problem of calculating the average conductance $\langle c\rangle$ of a quasi-one-dimensional disordered conductor can be mapped onto the problem posed by Eqs. (0.1) and (0.2). We are now in a position to solve the analogous but simpler problem that is obtained by taking for $\mathbf{G} / \mathbf{K}$ the hyperbolic superplane. More precisely, our goal is to solve the following problem [16]. Let $\gamma$ be a positive real parameter. Then, given the initial condition

$$
f_{\gamma}(g \cdot o ; s=0)=2^{1 / 2} \gamma\left(g g^{\dagger}\right)_{10} e^{-\gamma\left(\operatorname{str} g g^{\dagger}-1\right) / 4},
$$

we wish to solve the differential equation $(0.1)$ and evaluate the correlation function

$$
\langle c\rangle=\lim _{\gamma \rightarrow \infty} \int_{\mathbf{G} / \mathbf{K}} D g_{\mathbf{K}} f_{\gamma}(g \cdot o ; 0) f_{\gamma}(g \cdot o ; \beta) .
$$

Here $\beta$ is the length of the disordered conductor measured in units of the localization length, and the limit $\gamma \rightarrow \infty$ corresponds to the (realistic) case of a large number of scattering channels [8]. Note also that $\bar{f}_{\gamma}=f_{\gamma}$.

The problem is solved by using the Fourier transform (4.5) and its inverse (4.6). Strictly speaking, the application of Theorem 2 to $f_{\gamma}$ is not rigorous since, as it stands, the theorem has been proved only for functions with compact support. Without proof we will assume that, just as in classical analysis, the theorem can be extended to functions with sufficiently rapid decay at infinity. The function $f_{\gamma}(g \cdot o ; 0)$ defined in (5.1a) surely belongs to this class of functions, and it satisfies $\int_{\mathbf{G} / \mathbf{K}}^{\gamma} D g_{\mathbf{K}} f_{\gamma}(g \cdot o ; 0)=0$, since it is odd under the Cartan involution $g \cdot o \mapsto \hat{\theta} g \cdot o$.

Expressing $f_{\gamma}(g \cdot \sigma ; 0)$ in terms of its Fourier transform,

$$
f_{\gamma}(g \cdot o ; 0)=\int_{\mathbb{R}^{+}}\left(\int_{\mathbf{K} / \mathbf{M}} D k_{\mathbf{M}} \tilde{f}_{\gamma}(\lambda, k ; 0) e^{(-1 / 2+i \lambda) t\left(k^{-1} g\right)}\right) d \mu(\lambda),
$$

inserting (5.2) into (5.1b), and performing the integral over $\mathbf{G} / \mathbf{K}$, we obtain

$$
\langle c\rangle=\lim _{\gamma \rightarrow \infty} \int_{\mathbb{R}^{+}}\left(\int_{\mathbf{K} / \mathbf{M}} D k_{\mathbf{M}} \tilde{f}_{\gamma}(\lambda, k ; 0) \tilde{f}_{\gamma}(-\lambda, k ; \beta)\right) d \mu(\lambda) .
$$

Equation (0.1) is trivially solved by transforming to Fourier space and, in particular,

$$
\tilde{f}_{\gamma}(\lambda, k ; \beta)=e^{-\beta\left(\lambda^{2}+1 / 4\right)} \tilde{f}_{\gamma}(\lambda, k ; 0) .
$$

We must now evaluate

$$
\tilde{f}_{\gamma}(\lambda, k ; 0)=\int_{\mathbf{G} / \mathbf{K}} D g_{\mathbf{K}} e^{(-1 / 2-i \lambda) t\left(k^{-1} g\right)} f_{\gamma}(g \cdot o ; 0)
$$

To do that we introduce, in addition to $f_{\gamma, 1}(g \cdot o) \stackrel{\text { def }}{=} f_{\gamma}(g \cdot o ; 0)$, the functions $f_{\gamma, a}(g \cdot o)=2^{1 / 2} \gamma\left(g g^{\dagger}\right)_{a 0} e^{-\gamma\left(\operatorname{str} g g^{\dagger}-1\right)}$ for $a=2,3,4$. These transform as the fundamental representation of $\mathbf{K}$, and we thus get

$$
\tilde{f}_{\gamma, 1}(\lambda, k)=\int_{\mathbf{G} / \mathbf{K}} D g_{\mathbf{K}} e^{(-1 / 2-i \lambda) t(g)} f_{\gamma, 1}(k g \cdot o)=\sum_{a=1}^{4} k_{1 a} \tilde{f}_{\gamma, a}(\lambda, e),
$$

where $k_{1 a}$ are the elements in the first row of the matrix $k$, which are invariant 
under multiplication of $k$ from the right with $\mathbf{M}$, as they should be. The functions $\widetilde{f}_{\gamma, a}(\lambda, e)$ tend to a definite limit as $\gamma \rightarrow \infty$. By expressing $g g^{\dagger}$ in terms of the coordinates (1.2),

$$
\left(g g^{\dagger}\right)(v)=\left(\begin{array}{cc}
\left(1+v^{\dagger} v\right)\left(1-v^{\dagger} v\right)^{-1} & 2 v^{\dagger}\left(1-v v^{\dagger}\right)^{-1} \\
2 v\left(1-v^{\dagger} v\right)^{-1} & \left(1+v v^{\dagger}\right)\left(1-v v^{\dagger}\right)^{-1}
\end{array}\right)
$$

we find

$$
\lim _{\gamma \rightarrow \infty} \tilde{f}_{\gamma, a}(\lambda, e)=\lim _{\gamma \rightarrow \infty} 2^{3 / 2} \gamma \int_{\mathbf{D}} D v e^{(-1 / 2-i \lambda) v_{1}} v_{a} e^{-\gamma v^{\dagger} v}=2^{1 / 2}(-1 / 2-i \lambda) \delta_{a 1} .
$$

Using the coordinates $\varphi, \bar{\eta}$, and $\eta$ introduced in the proof of Theorem 1 , we finally evaluate the integral over $\mathbf{K} / \mathbf{M}$,

$$
\int_{\mathbf{K} / \mathbf{M}} D k_{\mathbf{M}}\left(k_{11}\right)^{2}=\frac{1}{2 \pi} \int_{0}^{2 \pi} \partial_{\bar{\eta}} \partial_{\eta}\left(\cos ^{2} \varphi\right)(1-2 \bar{\eta} \eta) d \varphi=1
$$

and thus obtain

$$
\langle c\rangle=2 \int_{\mathbb{R}^{+}} e^{-\beta\left(\lambda^{2}+1 / 4\right)}\left(\lambda^{2}+1 / 4\right) d \mu(\lambda)=2 \int_{0}^{\infty} e^{-\beta\left(\lambda^{2}+1 / 4\right)} \lambda \tanh (\pi \lambda) d \lambda .
$$

This is the result quoted in the introduction, where a discussion of the limits $\beta \rightarrow 0$ and $\beta \rightarrow \infty$ was given.

\section{Discussion}

In this work the Fourier transform for a rank-one noncompact homogeneous perfectly graded superspace $\mathbf{G} / \mathbf{K}$, the hyperbolic superplane, has been described. Our main result is Theorem 2 which, together with the corollary following it, establishes the invertibility of the Fourier transform for functions $f \in \mathscr{D}(\mathbf{G} / \mathbf{K})$. While admittedly concerning a rather special supermanifold, the theorem and its proof are, in the author's opinion, likely to be generalizable to Efetov's super coset spaces, thereby bringing within reach a variety of rigorous applications to quasi-onedimensional disordered systems.

It is instructive to compare the Fourier transform for the hyperbolic superplane with that of its classical partners, the hyperbolic spaces $H_{2 \rho+1}$ for $\rho=1 / 2,3 / 2,5 / 2$, etc. It turns out that many results for the hyperbolic superplane can be guessed by simply performing an analytical continuation to $\rho=-1 / 2$. (This is not unexpected since $H_{2 \rho+1}$ has dimension $2 \rho+1$ and a perfectly graded supermanifold behaves in many respects as a space of "effective" dimension zero.) For our purposes, the most important example is the formula for Harish-Chandra's c-function on the spaces $H_{2 \rho+1}$ [7],

$$
\mathbf{c}(\lambda)=2^{2 \rho-1} \pi^{-1 / 2} \Gamma(\rho+1 / 2) \frac{\Gamma(i \lambda)}{\Gamma(\rho+i \lambda)},
$$

valid for $\rho=1 / 2,1,3 / 2$, etc. Equation (3.5) can be obtained from this classical result by dropping the normalization factor $\Gamma(\rho+1 / 2)$ and then setting $\rho=-1 / 2$. Of course, in the process of doing so, one has to abandon the standard normalization 
convention $\mathbf{c}(-i \rho)=1$, since now $\mathbf{c}(-i \rho)_{\mid \rho=-1 / 2}=\mathbf{c}(i / 2)=0$, by the pole of $\Gamma(-1 / 2+i \lambda)$ at $\lambda=i / 2$. The zero of $\mathbf{c}(\lambda)$ at $\lambda=i / 2$ reflects the fact that

$$
\int_{\mathbf{K} / \mathbf{M}} D k_{\mathbf{M}}=0
$$

instead of being normalizable to unity. Another immediate consequence forced by (6.1) is the vanishing of the spherical functions $\phi_{\lambda}$ at the origin:

$$
\phi_{\lambda}(e)=0 .
$$

This gives rise to several modifications in comparison with the theory of the Fourier transform for the classical spaces $H_{2 \rho+1}$. The most substantial of these occurs in the proof of the analog of Theorem 2 where, for $H_{2 \rho+1}$, one first shows [7] that the identity

$$
f(o)=\int_{\mathbb{R}^{+}} \tilde{f}(\lambda) \phi_{\lambda}(e) d \mu(\lambda)
$$

holds for K-radial functions $f \in \mathscr{D}\left(H_{2 \rho+1}\right)$. For the hyperbolic superplane, however, this equation is correct only if $f(o)=0$, in which case it is downgraded by (6.2) to a triviality from which nothing can be deduced. A novel strategy in the proof of the Fourier inversion theorem is therefore called for, and the author has explored two alternatives.

One possibility is to continue to work with $\mathbf{K}$-radial $f$ and take a sufficient number of derivatives of Eq. (4.6) before setting $g=e$. Such an approach is eventually successful, but it involves manipulations with the kernel of the inverse of the Laplace-Beltrami operator and is somewhat indirect. The second approach, presented here, is murch better adapted in that it takes advantage of two of the features of the hyperbolic superplane that have no counterpart in classical geometric analysis. These are:

(A) Theorem 1, concerning the change of integration variables which is suggested by the diffeomorphism taking $(\mathbf{K} / \mathbf{M}) \times \mathbf{A}^{+}$to $\mathbf{G} / \mathbf{K}$;

(B) the fact that $\lim _{B \rightarrow \infty} \int_{0}^{B} \phi_{\lambda}\left(\exp r H_{0}\right) d \mu(\lambda)$ exists and has a well-defined meaning as an ordinary function (rather than as a distribution) for $r \neq 0$.

The integral for $B \rightarrow \infty$ was evaluated by closing the integration contour and using Cauchy's Theorem. The result is nonzero because $\mathbf{c}(\lambda)$, instead of being regular in the entire lower half of the complex $\lambda$-plane, has a simple zero at $\lambda=-i / 2$. All other ingredients used in the proof of Theorem 2, such as formula (3.2) and the expansion of the spherical functions in exponential functions, are standard.

Imagine now a more general homogeneous superspace $\mathbf{G} / \mathbf{K}$, still with the property that its base manifold is either a noncompact symmetric space, or a compact symmetric space, or the direct product of two such spaces as is the case for the super coset spaces of Efetov. Both (A) and (B) have generalizations to at least some supermanifolds of this kind, the crucial requirements being that they be perfectly graded and have no K-stable points other than the origin $o=e \mathbf{K}$. (This is what fuels the author's optimism concerning the validity of a more general version of Theorem 2.) In fact, although it is not evident from the proof, which was intended to be as "down-to-earth" as possible, the additional term $f(o)$ on the right-hand side of (2.4) is a consequence of no more than the K-stability of $o$ 
and the fact that its tangent space has "effective" dimension $p-q=0$. Specializing to the case of $\mathbf{K}$-radial $f$, and combining Theorem 1 with the rules of Berezin integration, we immediately conclude

$$
\int_{\mathbf{G} / \mathbf{K}} D g_{\mathbf{K}} f(g \cdot o)=f(o)
$$

since $f(k a \cdot o)=f(a \cdot o)$ does not contain any dependence on the anticommuting coordinates. Integral identities for invariant functions of this kind have been called the "Parisi-Sourlas-Wegner supersymmetric integral formula" in ref. [4]. As for (B), we observe that the Plancherel measure $d \mu(\lambda)$ given by Lemma 3 together with (3.6) contracts to the scale-invariant form $\lambda^{-1} d \lambda$ as $\lambda \rightarrow \infty$. Scale invariance at infinity is expected to hold for perfectly graded supermanifolds of the specified kind in general, for "large wave numbers probe short distances only." Simply put, $d \mu(\lambda)$ is $\lambda^{-1} d \lambda$ at $\lambda=\infty$ because $\delta\left(\exp r H_{0}\right) d r$ is $\underset{B}{r}-1 d r$ at $r=0$. Given the scale-invariant asymptotic form of $d \mu(\lambda)$, the integral $\int_{0} \phi_{\lambda}\left(\exp r H_{0}\right) d \mu(\lambda)$ for $r>0$ is rendered conditionally convergent in the limit $B \rightarrow \infty$ by the asymptotic behavior $\sim \lambda^{1 / 2} e^{i \lambda r}$ of the spherical functions $\phi_{\lambda}$ at $\lambda=\infty$. Clearly, similar statements should also apply to compact homogeneous spaces, Plancherel integrals now being replaced by Plancherel sums.

Let us finally turn to the heat kernel, $W$, for the hyperbolic superplane. Although the application given in Sect. 5 does not make any direct use of $W$, for other purposes one may wish to know it in explicit form. It is easy to guess the spherical expansion of $W$ from the results of Sect. 4. Consider the function $W_{1}$ defined on $\mathbf{A}^{+} \times \mathbb{R}^{+}$by

$$
W_{1}(a ; s)=1+\int_{\mathbb{R}^{+}} e^{-s\left(\lambda^{2}+1 / 4\right)} \phi_{\lambda}(a) d \mu(\lambda) .
$$

Extending $W_{1}(\because s)$ to a function on $\mathbf{G}$ bi-invariant under $\mathbf{K}$, we know that for any $g \in \mathbf{G}$ the function $f(h \cdot o ; s) \stackrel{\text { def }}{=} W_{1}\left(g^{-1} h ; s\right)$ is a solution of the heat equation $(0.1)$, owing to the invariance of $\Delta_{\mathbf{G} / \mathbf{K}}$ under left translations in $\mathbf{G}$. We claim that $W_{1}$ coincides with the heat kernel $W$, by the following argument. It is reasonable to assume that the kernel $\delta_{\boldsymbol{R}}^{\prime}$ which acts on functions $f \in \mathscr{D}(\mathbf{G} / \mathbf{K})$ by

$$
\left(\delta_{R}^{\prime} f\right)(h \cdot o)=\lim _{s \downarrow 0} \int_{\mathbb{R}^{+}}\left(\int_{\mathbf{G} / \mathbf{K}} D g_{\mathbf{K}} \phi_{\lambda}\left(g^{-1} h\right) f(g \cdot o)\right) e^{-s\left(\lambda^{2}+1 / 4\right)} d \mu(\lambda)
$$

is identical with the kernel $\delta_{R}$ introduced in (4.2). The corollary to Lemma 4 then asserts that

$$
\lim _{s \downarrow 0} \int_{\mathbf{G} / \mathbf{K}} D g_{\mathbf{K}} W_{1}\left(g^{-1} h ; s\right) f(g \cdot o)=f(h \cdot o),
$$

and hence the function $f(h \cdot o ; s)=\int_{\mathbf{G} / \mathbf{K}} D g_{\mathbf{K}} W_{1}\left(g^{-1} h ; s\right) f_{0}(g \cdot o)$ solves the Cauchy problem $(0.1)$ with initial condition $f(g \cdot o ; 0)=f_{0}(g \cdot o)$. Therefore, $W_{1}=W$. We conclude with the remark that (6.4) can be brought into the form

$$
W\left(\exp r H_{0} ; s\right)=\frac{e^{-s / 4}}{\sqrt{2 \pi s}} \int_{r}^{\infty} \frac{\sinh u}{\sqrt{\cosh u-\cosh r}} \exp \left(-\frac{u^{2}}{4 s}\right) d u,
$$

by a sequence of transformations not here presented. 
Acknowledgements. This work was performed within the research program of the Sonderforschungsbereich 341, Köln-Aachen-Jülich. The author thanks A. Huffmann for many useful discussions and $\mathrm{K}$. Frahm for reading the manuscript.

\section{Appendix}

We give a glossary of symbols.

$\mathbb{C}^{p, q}$ : the graded complex linear space of dimension $(p, q)$.

$\mathscr{A}$ : the parity operator fixing the grading of $\mathbb{C}^{p, q}$.

$\operatorname{Mat}(p, q)$ : the (associative) algebra of complex $(p+q) \times(p+q)$ matrices representing linear transformations of $\mathbb{C}^{p, q}$.

$\dot{\Lambda}_{2 N}$ : the Grassmann algebra generated by $2 N$ anticommuting variables $\xi_{1}, \bar{\xi}_{1}, \ldots, \xi_{N}, \bar{\xi}_{N}$ over $\mathbb{C}$. (The index $2 N$ is usually omitted.)

${ }^{0} \Lambda$ : the even part of $\Lambda$.

${ }^{1} \Lambda$ : the odd part of $\Lambda$.

$\mathbb{C}^{p, q}(\Lambda)$ : the Grassmann envelope of $\mathbb{C}^{p, q}$. (It is the even part of the tensor product of $\mathbb{C}^{p, q}$ with $\Lambda$.)

$\operatorname{Mat}(p, q \mid \Lambda)$ : the Grassmann envelope of $\operatorname{Mat}(p, q)$.

str: the supertrace.

sdet: the superdeterminant.

$\operatorname{GMat}(p, q \mid \Lambda)$ : the group of elements $X \in \operatorname{Mat}(p, q \mid \Lambda)$ satisfying sdet $X \neq 0$.

$m$ : the operator that projects onto the numerical part (or "body") of an element $z \in^{0} \Lambda$ or, more generally, of an element $X \in \operatorname{Mat}(p, q \mid \Lambda)$.

We use an adjoint of the second kind,

$$
\overline{\left(\bar{\xi}_{k}\right)}=-\xi_{k}, \quad \overline{\xi_{k} \xi_{l}}=\bar{\xi}_{k} \bar{\xi}_{l}
$$

for elements of ${ }^{1} \Lambda$. (Please note that this differs from Berezin's definition [3] in that the ordering of a product of elements remains unchanged when the adjoint is taken!) We use a standard representation of $\mathbb{C}^{p, q}$ by complex vectors with $p+q$ components in which $\mathscr{A}$ acts as the diagonal matrix $\mathscr{A}=\operatorname{diag}\left(1_{p},-1_{q}\right) \cdot\left(1_{n}\right.$ stands for the $n \times n$ unit matrix.) Writing elements $X \in \operatorname{Mat}(p, q \mid \Lambda)$ in this standard representation by blocks as

$$
X=\left(\begin{array}{ll}
A & B \\
C & D
\end{array}\right)
$$

we define an operation of hermitian conjugation by

$$
X^{\dagger}=\left(\begin{array}{cc}
A^{\dagger} & C^{\dagger} \\
-B^{\dagger} & D^{\dagger}
\end{array}\right), \quad \text { where } \quad\left(A^{\dagger}\right)_{k l}=\bar{A}_{l k}, \quad \text { etc. }
$$

This operation satisfies $(X Y)^{\dagger}=Y^{\dagger} X^{\dagger}$ and $\left(X^{\dagger}\right)^{\dagger}=X$ for $X, Y \in \operatorname{Mat}(p, q \mid \Lambda)$.

\section{References}

1. Abramowitz, M., Stegun, I. A.: Handbook of Mathematical Functions. Washington: National Bureau of Standards 1964

2. Aoki, K.: Heat kernels and super determinants of Laplace operators on super Riemann surfaces. Commun. Math. Math. Phys. 117, 405-429 (1988) 
3. Berezin, F. A.: Introduction to superanalysis. Dordrecht: Reidel 1987

4. Constantinescu, F., de Groote, H. F.: The integral theorem for supersymmetric invariants. J. Math. Phys. 30, 981-992 (1989)

5. Efetov, K. B.: Supersymmetry and the theory of disordered metals. Adv. Phys. 32, 53-127 (1983)

6. Helgason, S.: Differential geometry, Lie groups, and symmetric spaces. New York: Academic • Press 1978

7. Helgason, S.: Groups and geometric analysis. Orlando: Academic Press 1984

8. Iida, S., Weidenmüller, H. A., Zuk, J. A.: Statistical scattering theory, the supersymmetry method and universal conductance fluctuations. Ann. Phys. 200, 219-270 (1990)

9. Mahaux, C., Weidenmüller, H. A.: Shell model approach to nuclear reactions. Amsterdam: North Holland 1969

10. Rothstein, M. J.: Integration on noncompact supermanifolds. Trans. Am. Math. Soc. 299, 387-396 (1987)

11. Schäfer, L., Wegner, F.: Disordered systems with $n$ orbitals per site: Lagrange formulation, hyperbolic symmetry, and Goldstone modes. Z. Phys. B38, 113-126 (1980)

12. Stone, A. D., Szafer, A.: What is measured when you measure a resistance? The Landauer formula revisited. IBM J. Res. Dev. 32, 384 (1988)

13. Verbaarschot, J. J. M., Weidenmüller, H. A., Zirnbauer, M. R.: Grassmann integration in stochastic quantum physics: The case of compound-nucleus scattering. Phys. Rep. 129, $367-438(1985)$

14. Wegner, F.: Disordered systems with $n$ orbitals per site: $n=\infty$ limit. Phys. Rev. B19, 783-792 (1979)

15. Wegner, F.: The mobility edge problem: Continuous symmetry and a conjecture. Z. Phys. B35, 207-210 (1979)

16. Zirnbauer, M. R.: Fourier inversion theorem in mesoscopic transport. Physica A167, 132-139 (1990)

Communicated by H. Araki 